\title{
A Localization Model based on Irregular Quadrilateral for Wireless Sensor networks
}

\author{
Fengjun Shang ${ }^{1}$,Hongxia $\mathrm{Gao}^{2}$, IEEE \\ ${ }^{1}$ College of Computer Science and Technology, Chongqing University of Posts and Telecommunications \\ Chongqing 400065, China \\ ${ }^{2}$ College of Computer Science and Technology, Chongqing University of Posts and Telecommunications \\ Chongqing 400065, China
}

\begin{abstract}
Localization is used in location-aware applications such as navigation, autonomous robotic movement, and asset tracking to position a moving object on a coordinate system. In this paper, we present a localization model based on the irregular quadrilateral in wireless sensor networks. Firstly, a quadrilateral-positioning unit is presented. It may prevent the unknown node falls into the external of positioning unit. Lastly, we use RSSI value to find the nearest reference sample nodes from unknown node, compared with the SBL localization algorithm, our algorithm improve the positioning accuracy. Theoretical analysis and simulation results show that the algorithm in wireless sensor networks and other location-based applications have a good effect.
\end{abstract}

Index Terms-Localization; RSSI; Irregular Quadrilateral; Location accuracy.

\section{INTRODUCTION}

$\mathrm{W}$ ireless sensor networks (WSNs) can be applied to many areas, such as military affairs, commerce, medical care, environmental monitoring, and have become a new research focus in computer and communication fields. Many applications of WSNs are based on sensor self-positioning, such as battlefield surveillance, environment monitoring, indoor user tracking and others, which depend on knowing the location of sensor nodes. Because of the constraint in size, power, and cost of sensor nodes, the investigation of efficient location algorithms which satisfy the basic accuracy requirement for WSNs meets new challenges.

Wireless sensor network (Wireless sensor network) is a kind of multi-hop wireless network. It consists of a large number of sensor nodes, which are miniature, low cost, low power consumption, and be deployed in monitoring area. This network aims at the realization of sensitive data acquisition, processing and transmission of the area be covered $^{[1]}$. however, accurate nodes location information in wireless sensor network is more and more important, not only in construction and maintenance, but also in tracking positioning and other applications, current localization algorithm can be divided into range-based positioning mechanism $^{[2,3]}$ and range-free positioning mechanism ${ }^{[4,5]}$, its criteria is whether to measure and calculate the distance actually among nodes. range-based positioning mechanism mainly has: based on the Time of Arrival (TOA), based on Time Difference of Arrival (TDOA), based on reach Angle of Arrival (AOA) ,based on the Received Signal Strength Indicator ${ }^{[6]}$ (RSSI). Range-free positioning mechanism mainly has: centroid algorithm, approximate point-in-triangulation test localization algorithm (APIT), DV-Hop algorithm and the convex programming algorithm, etc.

$\mathrm{SBL}^{[7]}$ (Sequence-Based Location) localization algorithm can obtain certain positioning accuracy, but due to it must establish $N^{4}$ positioning sequence table ( there are $\mathrm{N}$ anchor nodes in the localization space), and its time overhead of the whole localization process is mainly on the list establishment, list storage and list comparison, so it needs to be improved. Zhu Jian proposed FTML ${ }^{[8]}$ positioning model, which combined the concept of relative approach degree in fuzzy mathematics, partially optimize the localization algorithm. Although the positioning model is novel and feasible, has considerable positioning accuracy, the premise of nodes localization implementation is that all unknown nodes must be deployed in positioning unit (triangle in our text) inside, it does not solve the problem that what positioning means should be taken if nodes are the outside of the positioning unit. The fact is that unknown nodes falls into the outside of the positioning unit, the nodes estimated coordinates will have a greater bias than its exact location, so this positioning algorithm has some limitations.

\section{Division OF QUADRANGLE AREA}

In this article, we select top 4 nearest anchor nodes from the unknown node as the reference anchor nodes, and the quadrilateral formed by this four reference anchor nodes is seen as the positioning unit where unknown node exits. We determine that whether unknown node is in the internal or external of quadrilateral by the area constraint relationships. This section addresses the following issues: If an unknown node is in the internal of quadrilateral, how to select the reference sample node; if an unknown node is in the external of quadrilateral, how to locate the unknown node as lower positioning error as possible.

In Fig.2, node $\mathrm{P}$ is unknown node, A,B,C,D are all the nearest reference anchor nodes from node $P$, then the quadrilateral formed by A,B,C,D is the positioning unit in this article. $\mathrm{AC}$ and $\mathrm{BD}$ are two diagonals of the quadrilateral $\mathrm{ABCD}, \mathrm{O}$ is intersections of that two diagonals (which coordinate can be calculated), and be considered as a new point of reference samples. Through the following area constraints, we can determine an unknown node is in the internal or external of quadrilateral ABCD. 
$S_{A B C D}=S_{\triangle A B P}+S_{\triangle B C P}+S_{\triangle C D P}+S_{\triangle D A P} \Rightarrow$ Node $\mathrm{P}$ is inside the Quadrilateral ABCD;

$S_{\triangle D A O}=S_{\triangle D P A}+S_{\triangle A P O}+S_{\triangle O P D} \Rightarrow$ Node P is inside the triangle DAO.

If an unknown node is inside of the quadrilateral ABCD, as shown in Fig. 2, then we can take following steps to narrow the positioning unit:

Step 1. Judge whether node $\mathrm{P}$ is inside triangle $\mathrm{ABO}$, triangle $\mathrm{BCO}$, triangle $\mathrm{CDO}$ or triangle $\mathrm{DAO}$.

Step 2. If node $\mathrm{P}$ is in any triangle referred above, then get three new sample nodes by getting the midpoint of all three sides of the triangle, namely three midpoints.

Step 3. Make comparisons between the vector RSSI of node $\mathrm{P}$ and three midpoints that we get from the last step in relative phase and find there nodes which have the highest relative phase, that is, which are closest to node $\mathrm{P}$ as node $\mathrm{E}$, node $\mathrm{G}$ and node $\mathrm{D}$.

Find midpoints of the new triangle and then get the sample nodes which are closest to node $\mathrm{P}$ so that narrow the possible area a node may locate. Repeat this until node $\mathrm{P}$ is finally in triangle LIM, in Fig. 2.

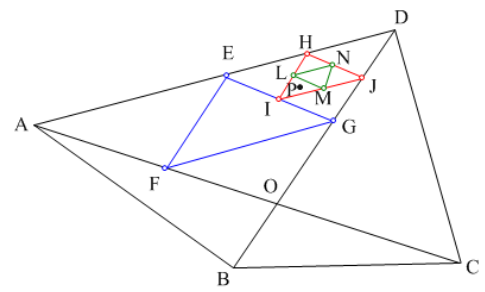

Fig. 2 internal Division of positioning Unit and determination of the sample points

If an unknown node is outside of the quadrilateral $\mathrm{ABCD}$, as shown in Fig. 3, we can determine the unknown node coordinates by two triangles that have common vertex.

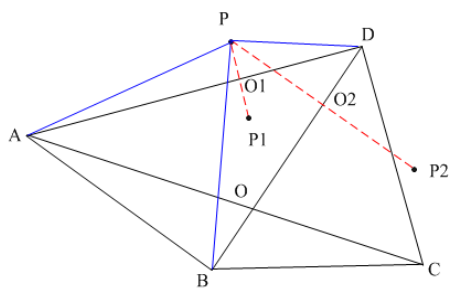

Fig. 3 Unknown nodes in the external of quadrilateral

Main steps are as follows:

Step 1. Find the top 2 highest value from RSSI vector(from strong to weak) of unknown node $P$, we can get node $\mathrm{D}$ and node $\mathrm{A}$. node $\mathrm{D}$, node $\mathrm{A}$ and node $\mathrm{P}$ can form a triangle named PAD.

Step 2. Find the top 1 and top 3 highest value from RSSI vector(from strong to weak) of unknown node $P$, we can get node $\mathrm{D}$ and node $\mathrm{B}$, node $\mathrm{D}$, node $\mathrm{B}$ and node $\mathrm{P}$ can form a triangle named PBD.

Step 3. As each triangle trilateral length is known, then the area of the triangle is determined; two vertex coordinates are known, with the formula for the area $S=\frac{1}{2} a \times h$, We can get the height from point $\mathrm{P}$ to its opposite side.

Repeat the operation of the step 3 above, respectively triangle $\mathrm{PAD}$ and triangle $\mathrm{PBD}$, we can get their common vertex coordinate, that the coordinate of node $\mathrm{P}$.

To sum up, when the unknown node is in internal of positioning unit, using a quadrilateral formed by four closest anchor node as new positioning unit, within the new positioning unit, constantly looking for the nearest reference sample point, so as to continuously reduce the region where unknown node exits; when the unknown node is in external of positioning unit, we take geometry and mathematical methods to estimate its coordinate.

\section{The Positioning MECHANism}

Theorem: If we take midpoint of each edge from a triangle, then this 3 new point and the 3 original apexes of the triangle are all called reference sample nodes, Each of this 6 reference sample nodes received signal strength Indicator from all anchor nodes is unique.

Proof: The proof is by contradiction. As shown in Fig.4. Assume that two different nodes P1 and P2 have different position, but they have same RSSI vector both in dimension and value (here the anchor node number is 3 , the vector dimension is 3$)$, Assume that $P 1(x, y)$, and the anchor node $\mathrm{P} 1$ to the anchor node $\mathrm{A}, \mathrm{B}, \mathrm{C}$ distance is respectively $d_{A 1} 、 d_{B 1}, d_{C 1}$. Corresponding, $\mathrm{P} 2$ also has $d_{A 2} 、 d_{B 2}$ 、 $d_{C 2}$.

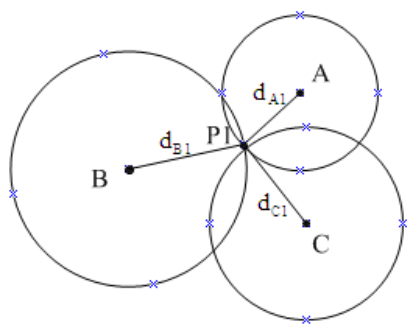

Fig.4. A group of RSSI vector uniquely corresponds to a node coordinates

$\because \quad \mathrm{P} 1$, P2 have same RSSI vector both in dimension and value.

$\Rightarrow$ Through the shadowing model ${ }^{[9]}, \mathrm{P} 1, \mathrm{P} 2$ have same distance vector both in dimension and value.

$\Rightarrow$ The distance that $\mathrm{P} 1$ to any anchor node is equal to that $\mathrm{P} 2$ to corresponding anchor node distance.

$\therefore$ There is the following equation:

$$
\left\{\begin{array}{l}
\sqrt{\left(x-x_{A}\right)^{2}+\left(y-y_{A}\right)^{2}}=d_{A 1} \\
\sqrt{\left(x-x_{B}\right)^{2}+\left(y-y_{B}\right)^{2}}=d_{B 1} \\
\sqrt{\left(x-x_{C}\right)^{2}+\left(y-y_{C}\right)^{2}}=d_{C 1}
\end{array}\right\}
$$




$$
\left(\begin{array}{l}
x \\
y
\end{array}\right)=\left(\begin{array}{l}
2\left(x_{A}-x_{C}\right) 2\left(y_{A}-y_{C}\right) \\
2\left(x_{B}-x_{C}\right) 2\left(y_{B}-y_{C}\right)
\end{array}\right)^{-1}\left(\begin{array}{l}
x_{A}^{2}-x_{C}^{2}+y_{A}^{2}-y_{C}^{2}+d_{C}^{2}-d_{A}^{2} \\
x_{B}^{2}-x_{C}^{2}+y_{B}^{2}-y_{C}^{2}+d_{C}^{2}-d_{B}^{2}
\end{array}\right)
$$

From above (1), (2), we can obtain the unique coordinates of the point $\mathrm{P} 1$.

And we have known assumptions:

$$
\left\{\begin{array}{l}
d_{A 1}=d_{A 2} \\
d_{B 1}=d_{B 2} \\
d_{C 1}=d_{C 2}
\end{array}\right\}
$$

Therefore, P2 and P1 have the same coordinates, and this contradiction with known assumptions, so original theorem is established. End of proof.

According to the theorem, each sample node in the subsequent location algorithm has a unique vector and it also ensures its uniqueness of coordinates.

In Fig.2, according to the quadrilateral area constraints, we can know that node $\mathrm{P}$ is within the quadrilateral ABCD. Through the triangle-area constraints, we can know that node $\mathrm{P}$ is within the triangle ACD. We take the positioning mechanisms that unknown nodes is within the quadrilateral, main steps is as follows:

Step 1. Triangle DAO has 6 reference sample nodes: D, A, O, E, F, G;

Step 2. Compare the RSSI vector similarity between node $\mathrm{P}$ and above 6 reference sample nodes;

Step 3. Find the nearest reference sample nodes by RSSI vector similarity, the most recent nodes in this paper is $\mathrm{E}, \mathrm{D}$, G.

Similarly, the nearest reference sample nodes from $\mathrm{P}$ in triangle EGD is node $\mathrm{H}$ - node I - node $\mathrm{J}$. Do the same operation to triangle HIJ, illustrating the node $\mathrm{P}$ is in the triangle LIM. So far, no longer narrow the micro-triangle region, triangle LIM shall be the final positioning unit, take the coordinates of triangle's centroid as the coordinates of node $\mathrm{P}$.

The location mechanism that unknown node falls outside of quadrilateral are as follows :

Figure 3, P is unknown node, A,B,C,D are all the nearest reference anchor nodes from node $\mathrm{P}, \mathrm{AC}$ and $\mathrm{BD}$ are two diagonals of the quadrilateral $A B C D$, point $O$ is intersections of that two diagonals (which coordinate can be calculated), and be considered as a new point of reference samples.

According to the quadrilateral area constraints, equality is not true; point $\mathrm{P}$ is outside of the quadrilateral ABCD. Unknown node $\mathrm{P}$ receives RSSI from four reference anchor nodes, and the RSSI vector ordered from strong to weak is:

$$
\left\{R S S I_{D}, R S S I_{A}, R S S I_{B}, R S S I_{C}\right\}
$$

Point $\mathrm{D}$, point $\mathrm{A}$ and point $\mathrm{P}$, composed of triangle PAD. Here, due to that RSSI value can be measured between every two points, by signal attenuation models, we can find each distance between every two points (three sides of a triangle). Because node $\mathrm{A}$ and node $\mathrm{D}$ are anchor nodes, so the coordinates of unknown node $\mathrm{P}$ can be obtained by using the following equation:

$$
\left\{\begin{array}{l}
L=d_{A D}+d_{D P}+d_{P A} \\
S_{\triangle P A D}=\sqrt{\frac{L}{2} *\left(\frac{L}{2}-d_{A D}\right) *\left(\frac{L}{2}-d_{D P}\right) *\left(\frac{L}{2}-d_{P A}\right)} \\
S_{\triangle P A D}=\frac{1}{2} d_{A D} * d_{P O 1} \\
d_{P O 1}=\frac{A * X+B * Y+C}{\sqrt{A^{2}+B^{2}}}
\end{array}\right\}
$$

Where $L$ is the perimeter of the triangle and $S$ is the triangular area, $d$ is the triangle side, equation $A^{*} X+B^{*} Y+C=0$ is the straight line that contains A and D. By solving systems of equations (4), we can get two sets of coordinates that can strike unknown node $\mathrm{P}: P(X, Y)$ and $P 1(X 1, Y 1)$.

Similarly, select the first value point $\mathrm{D}$ and third value point $\mathrm{B}$ in RSSI vector, together with the point $\mathrm{P}$ itself, can form triangle PBD and obtain two sets of unknown node coordinates: $P(X, Y)$ and $P 1(X 2, Y 2)$. Integrated solutions of two groups of two triangles, find their common vertex $P$, we can get the unknown node $P$ 's coordinates: $P(X, Y)$.

\section{Simulation Analyses}

We simulate our localization algorithm on matlab7.0 in this subsection, unknown node number UN is 160 , all nodes are homogeneous, every unknown node is randomly placed in an experiment environment with $10 \times 10 \mathrm{~m}^{2}$ square area and all the anchor nodes are within unknown communication range.

Table 1 shows each positioning an unknown node, comparison results of the number of reference sample nodes needed in our localization algorithm and FTLM model localization algorithm. From table 1, we can know that in the case of positioning micro-area is the same, our algorithm requires less number of reference sample nodes, and calculation complexity is significantly lower. Last, this iteration positioning by narrowing positioning area is convergent.

Table 1 Comparison of the number of reference sample nodes needed in our localization algorithm and LTFM model localization algorithm

\begin{tabular}{|l|l|l|l|}
\hline Algorithm & S/64 & S/256 & S/512 \\
\hline Our Algorithm & 13 & 16 & 19 \\
\hline FTLM & 13 & 16 & 37 \\
\hline
\end{tabular}

Also, in the targeted area of $10 \times 10 \mathrm{~m}^{2}$ square area, Fig. 5 show our algorithm performance by comparing our positioning algorithms and 3 centroid in triangle with sequence algorithm, SBL and 3 centroid in triangle algorithm.Fig. 5 shows their respective positioning errors. 


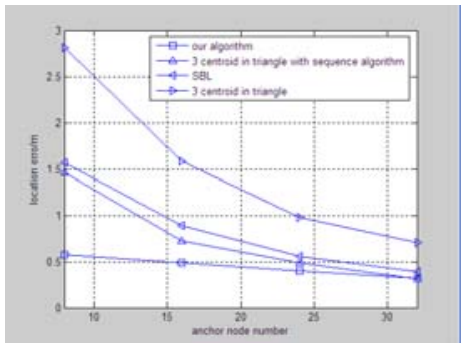

Fig. 7 Algorithm performance comparison between our localization algorithm and other localization methods

Thus, three methods of localization errors are all reduced with the increase number of anchor nodes, this is because the more anchor nodes, the original localization area is divided into different types of smaller regions, unknown nodes are closer to the anchor nodes and then the localization is more accurate. We can know that the localization error of " 3 centroid in triangle" algorithm is 5 times higher than our algorithm from Fig.7. This is because we continually narrow region area where unknown node exits to $1 / 4$ in our localization process, so as to estimate unknown node coordinate better. When the number of anchor nodes is 32, the localization of our location algorithm and other algorithms is almost equal, because the number of anchor nodes increase to 32, the number of sequence is up to 462273. $\left(\frac{n^{4}}{2}-2 \div n^{3}+\frac{7 * n^{2}}{2}-2 \times n+1,(n=32)\right.$ ).At this time, the region that unknown nodes exit is rather small, but this obviously needs to do a lot of work for computing and sequence alignment, and at this point. Each unknown node in our localization algorithm just need to compare with 16 reference sample nodes to estimate its own coordinate, but still has lower localization error, showing its superiority.

\section{Conclusions}

This paper proposed a novel localization algorithm based on RSSI vector similarity, at first, The method determine to adopt which positioning mechanism by judging unknown nodes in internal or external of the positioning unit. When unknown nodes in the internal of positioning area, by finding the midpoint of each edge of the triangle to narrow localization area; With the concept of RSSI value, determine the most 3 recent reference sample nodes by iteration, and take triangle centroid as the estimated coordinates of unknown node. This article also verifies the feasibility of algorithms and positioning accuracy through simulation and analysis. Our next study will optimize our algorithm to make it can work in a more complex environment well.

\section{ACKNOWLEDGEMENTS}

This research work funded by the Chongqing Education Commission for Scientific and Technological Research Projects(KJ110504), Chongqing Municipal Science and Technology Commission of Natural Science Fund
(cstc2012jjA40038). Thanks experts for your valuable comments.

\section{REFERENCES}

[1] Ren Fengyuan, Huang Haining, Lin Chuang. Wireless sensor network [J]. Journal of Software 2003, 14(7): 1282-1290

[2] Dai Gui-lan, Zhao Chongchong, Qiu Yan. A location scheme based on sphere for wireless sensor network in 3D [J]. Acta Electronica Sinica, 2008,36(7):1297-1303

[3] Awides A, Park H, Srivastava M B. The bits and flops of the N-hop multilateration primitive for node localization problems[A].Proc. of the 1st ACM Int. Workshop on Wireless Sensor Networks and Application[C]. Atlanta, 2002, 112-121

[4] Jianliang $\mathrm{Xu}$,Xueyan Tang, Wang Chein Lee. A new storage scheme for approximate location queries in object-tracking sensor networks [J].IEEE Transaction on Parallel and Distributed Systems, 2008, 19(2):262-275.

[5] Kevin Yuen,Ben Liang, Bacchum Li. A distributed framework for correlated data gathering in sensor networks[J].IEEE Transaction on Vehicular Technology, 2008,57(1):578-593

[6] Zhao zhao, Chen xiaohui. Improved positioning algorithm based on RSSI in wireless sensor networks [J]. Journal of Transduction Technology, 2009, 22(3): 391- 394

[7] Liu zhihua, Chen Jiaxing, Chen Xiaokai. A new algorithm research of Sequence-based localization technology in wireless sensor networks [J]. Journal of electronic, 2010, 38(7):1552-1556

[8] Zhu jian, zhao hai, xu jiuqiang. Localization Model in Wireless Sensor Networks [J]. Journal of Software, 2011, 22(7):1612-1625

[9] K Yedavalli, B krishnamachari. Sequence-Based Localization in Wireless Sensor Networks [J].IEEE Transcations on Mobil e Computing, 2008,7(1): 81- 9 